\title{
Comparing two types of navigational interfaces for Virtual Reality
}

\author{
Luís Teixeira ${ }^{\mathrm{a}, \mathrm{b}}$, Elisângela Vilar ${ }^{\mathrm{a}, \mathrm{b}}$, Emília Duarte ${ }^{\mathrm{b}, \mathrm{c}}$, Francisco Rebelo ${ }^{\mathrm{a}, \mathrm{b}^{*}}$ and Fernando Moreira \\ da Silva ${ }^{\mathrm{d}}$ \\ ${ }^{a}$ Ergonomics Laboratory, FMH/Technical University of Lisbon, Estrada da Costa, 1499-002 Cruz Quebrada- \\ Dafundo, Portugal. \\ ${ }^{\mathrm{b}}$ CIPER - Interdisciplinary Center for the Study of Human Performance, Technical University of Lisbon, Estrada \\ da Costa, 1499-002 Cruz Quebrada, Dafundo, Portugal \\ ${ }^{\mathrm{c}}$ UNIDCOM/IADE - Institute of Arts, Design and Marketing, Av. D. Carlos I, no. 4, 1200-649 Lisbon, Portugal. \\ ${ }^{\mathrm{d}}$ Art and Design Department- FA/Technical University of Lisbon, Rua Sá Nogueira, Alto da Ajuda 1349-055 \\ Lisbon, Portugal.
}

\begin{abstract}
Previous studies suggest significant differences between navigating virtual environments in a life-like walking manner (i.e., using treadmills or walk-in-place techniques) and virtual navigation (i.e., flying while really standing). The latter option, which usually involves hand-centric devices (e.g., joysticks), is the most common in Virtual Reality-based studies, mostly due to low costs, less space and technology demands. However, recently, new interaction devices, originally conceived for videogames have become available offering interesting potentialities for research. This study aimed to explore the potentialities of the Nintendo Wii Balance Board as a navigation interface in a Virtual Environment presented in an immersive Virtual Reality system. Comparing participants' performance while engaged in a simulated emergency egress allows determining the adequacy of such alternative navigation interface on the basis of empirical results. Forty university students participated in this study. Results show that participants were more efficient when performing navigation tasks using the Joystick than with the Balance Board. However there were no significantly differences in the behavioral compliance with exit signs. Therefore, this study suggests that, at least for tasks similar to the studied, the Balance Board have good potentiality to be used as a navigation interface for Virtual Reality systems.
\end{abstract}

Keywords: Virtual Reality, Navigation interfaces, Human performance, Emergency egress

\section{Introduction}

Such as object manipulation, the displacement between two points, being with purely exploratory goals or connected to the execution of a task, is one fundamental human capability, expected in any environment, even virtual ones. An unnatural, intrusive and cognitively demanding navigation can become an influential factor over the results of studies that use Virtual Reality (VR), compromising its validity.

Therefore, by its relevance, the navigation quality becomes a critical requirement for the interaction in Virtual Environments (VE), with a great impact in the quality of the experience. However, due to vari- ous reasons, many of them technological, the navigation in VEs is often quite limited, being away from what is the walking experience in the real world. For example, the lack of proprioceptive cues and energy consumption that are associated to walking in the real world, transforms the navigation in a kind of "sliding through the world" experience.

Among the more obvious consequences of this limitation is the reduction of the sense of presence (e.g., [16]) that, paradoxically, is promoted by the use of an immersive system (e.g. Head-Mounted Displays). Another notable consequence is the potential reduction of ecological validity since it affects the way the user moves [13], which can compromise

\footnotetext{
* Corresponding author. Email: frebelo@fmh.utl.pt
} 
some studies. In this regard, stands out for example, studies that assess the quality of wayfinding (e.g., $[3,4,7])$, studies that evaluate the human behavior during an emergency egress (e.g., $[5,10])$ or those that evaluate compliance with warnings (e.g., $[14,15])$.

There are several types of interfaces used for navigation in VEs. In generic terms, it is possible to classify interfaces in two groups: passive and active. The main characteristic, that allows differentiating between the groups, is the physical activity of the user. That is, with a passive interface (e.g., joystick) the user remains stationary, almost always in a seated position, without spending much energy. In contrast, the real movement of the user's body, spending more energy, is typical of active interfaces (e.g., treadmills, pedaling devices, step-in-place systems).

Previous studies suggest a trend towards better performance with active navigation devices. Change et al. [21] suggest that spatial orientation improves more when users are allowed to physically turn and translate than when they do it virtually. Bakker et al. [18] found that vestibular feedback increases the accuracy in determining the angle of the turn.

Reviewing the literature, it can be verified that the joystick has been one of the most used navigation interface in VR related studies. This popularity can be related not only with economic (i.e., low cost of the device), operational (i.e., off-the-shelf product) and technological aspects (i.e. ease of integration in the VR systems) but also with aspects related with the ease of use and the degree of familiarity of the users with these products. The familiarity of the users with the interface can affect the performance, as well as the required time for training, which is necessary to ensure that all the participants attain equal skill level in controlling the device.

Additionally, besides the already mentioned consequences, the hand-centric passive navigation interfaces, also usually limit the manipulation of objects in VEs, because the hand(s) are used for the navigation.

To try to overcome these limitations, other types of active interfaces have been developed for navigation in VEs. Some solutions use the lower-body to control the movement (e.g., $[1,9])$ and others use the complete body (e.g., [16]).

These interfaces, despite the promising results, are not still in a commercial form, or in some cases (e.g., $[6,8,19])$ place demands that are impractical for many laboratories such as large space for the device and frequent, specialized maintenance with high costs.
In this context, recently there is an increase of interest, from researchers, in the use of devices developed for the gaming industry. These interfaces, available at accessible costs, offer interesting potentialities to be used in VR systems as innovative ways to interact with VEs.

The Nintendo ${ }^{\circledR}$ Wii Balance Board (Balance Board hereafter), is one of those examples that can use the shift of weight over it to control the navigation in a VE. Although it is not, definitely, an active navigation interface, the Balance Board presents some characteristics that approximates it from that group, namely due to the increase of energy spending and the upright position of the user. Another advantage is that it frees the user's hands for a greater possibility of object manipulation inside the VE.

This exploratory study had as its main objective to explore the potentialities of the Balance Board as an alternative navigation interface in VEs. It was compared the performance of participants in an emergency egress in a VE, using a passive interface (i.e., Joystick) versus using the Balance Board.

The following variables were measured for the comparison: compliance with the exit signs, time spent and distance travelled in the simulation.

The choice of an emergency egress as a study task, is mainly supported by the increased cognitive demands for the participants and that will promote the evaluation of the real impact of the interface on the success of the task, since the effect of stress in human performance is well known, namely in visual attention, decision making and motor control (e.g., $[2,12])$, even in routine tasks. Therefore, it is expected that the performance decreases in navigation interfaces that place more cognitive demands to the user.

The results of this study can be useful to support the decision to continue to develop processes to integrate the Balance Board, as an alternative navigation interface for VEs, in immersive VR systems.

\section{Method}

\subsection{Study's design and protocol}

The study used a between-subject design with two experimental conditions (Balance Board and Joystick). The experimental conditions were compared through a search task in a VE using the following performance variables: Time spent, Distance travelled and Behavioral compliance with exit signs. 
Time spent is the time, in seconds, that the participant takes to complete the simulation. Distance travelled is the total distance, in meters, that the participant travelled in the simulation. Behavioral compliance is given by the number of correct choices at the T-shaped intersections, at the first attempt, that is, turns towards the direction indicated by the exit sign.

The experimental session was divided in four stages: a) introduction to the study; b) training; c) simulation; and d) follow-up interview.

a) The experimental session started with the signing of a consent form and participants were instructed that they could end the experiment at any time. After that, they were introduced about the study and the equipment that they would be using to interact in the simulation. To reduce the possibility that participants could deliberately try to perform better with a specific navigation interface, participants were told that the main objective of the study was to validate new VR software, being developed at the laboratory, which automatically captures human interaction's data.

b) The training stage consisted in placing the participants in a training VE so they could familiarize themselves with the equipment. The VE was a small room with a pillar in the center and a connection to a zigzag type of corridor. Participants were asked to explore the VE freely until they felt able to control the navigational interface. At that time, the researcher asked the participants to go around the pillar in both directions and to go up to the end of the corridor. If the participants could fulfill these goals without difficulties, the researcher would start the simulation stage.

c) In the simulation stage, participants were told that they were in an emergency egress and they had to get out of a building (described in topic 2.3) in the minimum amount of time possible. During the simulation there was a fire alarm sound that played for the duration of the simulation. The simulation ended when the participant reached the end point of the building, if the participants stated that wanted to stop the simulation or after 10 minutes (stopped by the researcher).

d) After the simulation, participants engaged in an exploratory free-style follow-up interview regarding the difficulties that they experienced in the simulation and their opinion regarding the navigation interface used.

\subsection{Sample}

Forty university students participated in this study, equally distributed in gender and number (20 males and 20 females) by the two experimental conditions.

Participants had between 19 and 34 years old for the Joystick condition (mean $=22.9, \mathrm{SD}=3.32)$ and between 18 and 29 years old for the Balance Board condition $($ mean $=21.10, \mathrm{SD}=3.11)$.

The criteria used for the acceptance of participants was: participants had to be university students, between 18 and 35 years old, fluent in Portuguese, no color vision deficiencies (verified by the Ishihara Test [20]), could not use glasses (corrective lenses allowed) because of a characteristic of the HMD, that did not allow the use of glasses. Also, participants had no problems that could prevent them from participating or would be aggravated by the participation in the experiment (reported by them).

\subsection{Virtual Environment}

The VE was created with the emergency egress in mind. As such, the VE was designed as a set of six T-shaped intersections, where only one option (left/right) provides access to the next exit and the other option presented a closed door (see Figure 1).

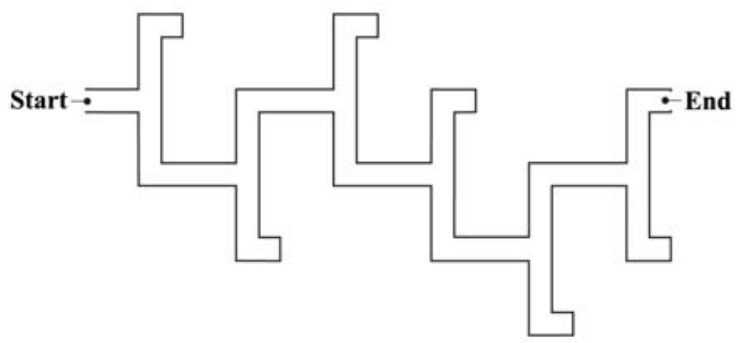

Figure 1 - Top view of the Virtual Environment

Each intersection was marked by a capital letter label, so participants would not feel disoriented, thinking that they were always returning to the same location. The exit signs were placed at each intersection to mark the routes of egress and their dimensions were 30 by $15 \mathrm{~cm}$ and were placed in the wall at $2.20 \mathrm{~m}$ from the floor.

The VE was modeled in Autodesk 3dsMax v2009 and exported through the plugin OgreMax v1.6.23 to be presented by the ErgoVR system [11]. 


\subsection{Equipment}

The equipment used was: (a) A magnetic motion tracker from Ascension-Tech $\AA$, model Flock of Birds, with 6DOF, used for the motion detection of the head; (b) Head-Mounted Display from Sony ${ }^{\circledR}$, model PLM-S700E; (c) Wireless headphones from Sony ${ }^{\circledR}$, model MDR-RF800RK; (d) Graphics Workstation with an Intel ${ }^{\circledR}$ i7 processor, 8 Gigabytes of RAM and a nVIDIA ${ }^{\circledR}$ QuadroFX4600.

The only difference between the conditions was the navigation interface used, that is, the Nintendo ${ }^{\circledR}$ Wii Balance Board and a Thrustmaster ${ }^{\circledR}$ USB Joystick.

\subsection{Navigation}

In this study, two types of navigation interfaces were used, a Balance Board and a Joystick.

The Balance Board is a device that has four pressure sensors, one on each corner (see Figure 2). This device gives the weight detected from each sensor and also the center of balance. The center of balance is the projection of the center of mass on the platform.

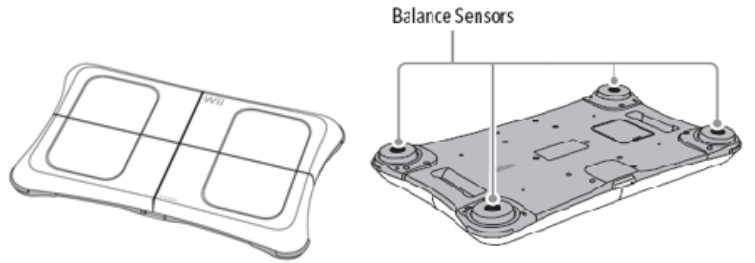

Figure 2 - Balance Board (up-side down view on the right)

The movement in the VE is made by shifting more weight to the desired direction in the Balance Board, that is, if someone wants to move forward, it should apply more weight on the two sensors in the front. If someone wants to change direction in the VE, it should apply more weight on the two sensors on the left or right side. That movement is represented in the $\mathrm{VE}$ as a rotation of the virtual body of the person to the desired direction. Also, the more weight applied, the faster the corresponding movement.

The response of the Joystick in the simulation is identical, that is, if someone moves the Joystick forward, that represents a forward movement in the VE, and if someone moves the Joystick to the left or to the right, it represents a rotation of the virtual body to the desired direction, just like it happens in the Balance Board. Also, the movement speed is directly proportional to the way that the Joystick is tilted.

\section{Results}

The statistical analysis, performed in IBM ${ }^{\circledR}$ SPSS $®$ Statistics v19, was conducted at a $5 \%$ significance level.

For this study, the dependent variables assessed, which were collected automatically by the ErgoVR system were: Time spent, Distance travelled and Behavioral compliance with exit signs.

The Wilcoxon-Mann-Whitney test was used to evaluate behavioral compliance differences between the experimental conditions (see Figure 3), i.e. number of times the participants went correctly in the direction indicated by the exit sign. Results $(\mathrm{U}=173$; $\mathrm{W}=383 ; p=.461)$ show that there were no significant differences between the Joystick users $(\mathrm{mdn}=5.0)$ and the Balance Board users $(\mathrm{mdn}=5.0)$.

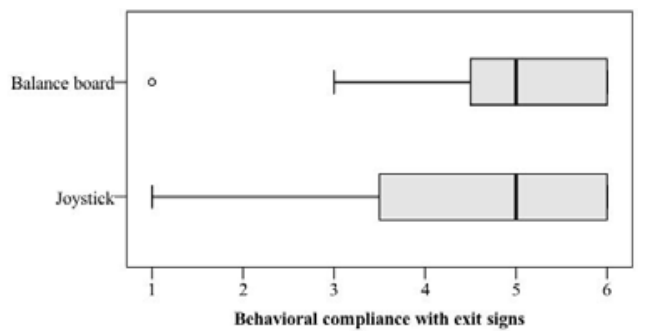

Figure 3 - Boxplot for the behavioral compliance with exit signs

Two non-parametric Analysis of Covariance (ANCOVA) tested the differences regarding time spent and distance travelled between the experimental conditions, after controlling for the effect of behavioral compliance.

Results from the non-parametric ANCOVA show that the time spent (see Figure 4) by the Joystick users in the simulation $(\mathrm{mdn}=84.48 \mathrm{~s}$ ) was significantly lower than for the Balance Board users $(\mathrm{mdn}=147.15 \mathrm{~s})$, after removing the effect of the behavioral compliance, with $\mathrm{F}(1,38)=44.585$ and $p<.001$.

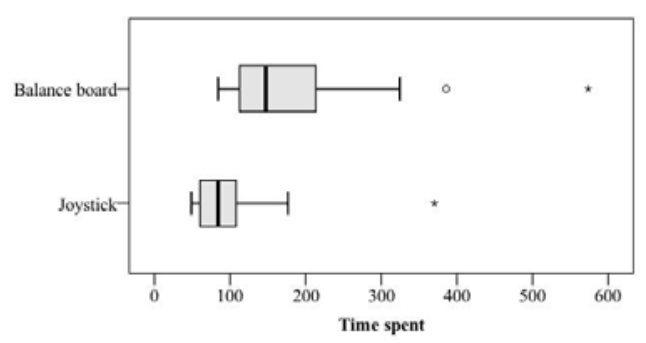

Figure 4 - Boxplot for Time Spent 
Regarding the distance travelled (see Figure 5), results from the non-parametric ANCOVA show that Joystick users $(\mathrm{mdn}=199.61 \mathrm{~m})$ travelled significantly less distance than the Balance Board users $(\mathrm{mdn}=461.67 \mathrm{~m})$, after removing the effect of the behavioral compliance, with $\mathrm{F}(1,38)=64.961$ and $p<.001$.

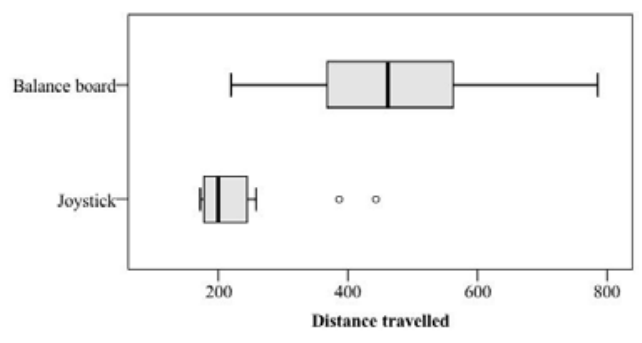

Figure 5 - Boxplot for Distance travelled

\section{Discussion and Conclusion}

Diverse interfaces can be used to allow participants to navigate in a VE. Those interfaces can differ regarding the degree they replicate the real human walking. In spite of the interest about this subject, there are few empirical comparisons reported in literature involving devices originally developed for the game industry.

In this context, this exploratory study aimed to investigate the differences between navigating using a common joystick and using the Balance Board, while performing an emergency egress in a VE. For such, several measurements were compared: compliance with the exit signs, time spent and distance travelled in the simulation.

The results show no significant differences on compliance between participants using both devices. However, statistical significant differences exist between the two evaluated interfaces, in the time spent and distance travelled in the simulation.

These results suggest that, although exerting influence on the participants' navigation ability, by making them travel different distances with different velocities, the devices did not influenced, in a significant manner, their ability to accomplish the given task (i.e., find the way out of the VE).

The higher values (worse performance) in time spent and distance travelled in the Balance Board users might be connected to the navigation style adopted. According to the informal opinions from the participants, the rotation movement was somehow cumbersome and sometimes they had to "think" be- fore moving. This might happened as a result of a lower adaptation time than they probably required. However, the use of the Balance Board showed more enthusiasm from the participants than the use of a Joystick.

For future work, we can see different paths at the moment. One is the improvement of the rotational movement that got the worst critics from the participants, mainly because they had to change directions rapidly in somehow confined spaces and the participants had more trouble to do so with the Balance Board than with the Joystick.

Another path is to improve the training moment for the Balance Board, in order to be less subjective, with pre-defined criteria and navigational tasks that could be automatically analyzed and therefore only allow passing to the simulation stage when the participants fulfill those training tasks.

Future work should also assess the impact of navigation interfaces in the sense of presence and simulator-sickness.

The present research shows that for VR-based studies, where performance variables such as time spent and distance travelled are not important, the use of the Balance Board (created as a game interface) is a viable option.

\section{References}

[1] B. Peterson, M. Wells and T. Furness. The effects of the interface on navigation in virtual environments. In Proceedings of Human Factors and Ergonomics Society 1998 Annual Meeting, 1998.

[2] C. D. Wickens and J. G. Hollands. Engineering psychology and human performance. Prentice Hall, 2005.

[3] E. Cubukcu. Relation of physical form to spatial knowledge in largescale virtual environments. Environment and Behavior, 37 (2005), 397-417.

[4] E. Vilar, F. Rebelo and L. Teixeira. The influence of guidance system in indoor wayfinding using virtual reality. In Proceedings of the First International Conference on Integration of Design, Engineering and Management for innovation IDEMI09 (in CD-ROM form), Porto, Portugal, 2009.

[5] G. Mantovani, L. Gamberini, M. Martinelli and D. Varotto. Exploring the suitability of virtual environments for safety training: Signals, norms and ambiguity in a simulated emergency escape. Cognition, Technology \& Work, 3(2001), 33-41.

[6] H. Iwata and T. Fuji. Virtual perambulator: A novel interface device for locomotion in virtual environment. Virtual Reality, (1996), 60-65.

[7] I. Omer and R. Goldblatt. The implications of inter-visibility between landmarks on wayfinding performance: An investigation using a virtual urban environment. Computers, Environment and Urban Systems, 31 (2007), 520-534.

[8] J. Hollerbach, D. Grow and C. Parker. Developments in locomotion interfaces. 9th International Conference on Rehabilitation Robotics, ICORR2005, 522-525. IEEE, 2005. 
[9] L. Bouguila, E. Florian and M. Courant. Active walking interface for human-scale virtual environment. 11th International Conference on Human-Computer Interaction, HCII'05. 22-27 July 2005, Las Vegas, Nevada, USA, 2005.

[10]L. Gamberini, P. Cottone, A. Spagnolli, D. Varotto and G. Mantovani. Responding to a fire emergency in a virtual environment: Different patterns of action for different situations. Ergonomics, 46 (2003) 842-858.

[11]L. Teixeira, F. Rebelo and E. Filgueiras. Human interaction data acquisition software for virtual reality: A user-centered design approach. In D. B. Kaber \& G. Boy, eds., Advances in Cognitive Ergonomics. Advances in Human Factors and Ergonomics Series, CRC Press/Taylor \& Francis, Ltd, Miami, Florida, 2010, pp. 793-801.

[12]M. A. Staal. Stress, cognition, and human performance: A literature review and conceptual framework. NASA/JSC-TM2004-212824. NASA Johnson Space Center, Houston, 2004

[13] M. C. Whitton, J. V. Cohn, J. Feasel, P. Zimmons, S. Razzaque, S. J. Poulton, B. McLeod et al. Comparing VE locomotion interfaces. In IEEE Proceedings Virtual Reality 2005, 123-130. IEEE, 2005

[14]M. E. C. Duarte, F. Rebelo and M. S. Wogalter. Virtual reality and its potential for evaluating warning compliance. Human Factors and Ergonomics in Manufacturing \& Service Industries, 20 (2010), 526-537.

[15]M. E. C. Duarte and F. Rebelo. Virtual reality in the study of warnings effectiveness. In M. J. Dainoff, ed., Ergonomics and Health Aspects of Work with Computers. International Conference, EHAWC 2007, Held as Part of HCI International 2007
Beijing, China, July 2007, Proceedings. Lecture Notes In Computer Science 4566, Springer-Verlag Berlin Heidelberg, New York, 2007, pp. 189-198.

[16]M. Slater, M. Usoh and A. Steed. Taking steps: The influence of a walking technique on presence in virtual reality. ACM Transactions on Computer-Human Interaction, 2 (1995), 201-219.

[17]M. Usoh, K. Arthur, M. C. Whitton, R. Bastos, A. Steed, M. Slater and F. P. Brooks. Walking $>$ walking-in-place $>$ flying, in virtual environments. In Proceedings of the 26th annual conference on Computer graphics and interactive techniques SIGGRAPH '99, ACM Press, New York, 1999, pp. 359-364.

[18]N. H. Bakker, P. J. Werkhoven and P. O. Passenier. Aiding orientation performance in virtual environments with proprioceptive feedback. In Proceedings of the Virtual Reality Annual International Symposium, IEEE Computer Society, Washington, DC, 1998, (p. 28).

[19] R. P. Darken, W. R. Cockayne and D. Carmein. The omnidirectional treadmill : A locomotion device for virtual worlds In Proceedings of UIST '97, Banff, Canada, 1997, pp. 213-221.

[20]S. Ishihara. Tests for colour-blindness, 38 plates edition. Tokyo, Kanehara \& Co. LTD, Japan, 1998.

[21] S. S. Chance, F. Gaunet, A. C. Beall and J. M. Loomis. Locomotion mode affects the updating of objects encountered during travel: The contribution of vestibular and proprioceptive inputs to path integration. Presence: Teleoperators and Virtual Environments, 7 (1998), 168-178. 